\title{
Erratum to: Multiple Scattering of Seismic Waves from Ensembles of Upwardly Lossy Thin Flux Tubes
}

\author{
Chris S. Hanson ${ }^{1}$ Paul S. Cally ${ }^{1,2}$
}

Published online: 21 January 2016

(C) Springer Science+Business Media Dordrecht 2016

\section{Erratum to: Solar Phys (2015) 290: 1889-1896 \\ DOI 10.1007/s11207-015-0732-x}

The wording in the caption of Figure 5 is in conflict with the rest of the text. Specifically, the incorrect caption indicates a result that is the opposite of what is presented in the rest of the article. We apologise for this mistake. The caption should be changed to the following:

The absorption (left) and phase shift (degrees, right) of the $m=0$ (top) and $m=1$ (bottom) incident and outgoing $f$-mode waves interacting with a random ensemble of growing tube number of identical properties ( $\beta=1$ : filled-black circles; $\beta=0.1$ : filled-red triangles; $\beta=$ 10: filled-green squares) with the radiative (symbols only) and stress-free (symbols with lines) boundary conditions.

The results and the text of the main body remain correct.

The online version of the original article can be found under doi:10.1007/s11207-015-0732-x.

P.S. Cally

paul.cally@monash.edu

C.S. Hanson

hanson@mps.mpg.de

1 Max-Planck-Institut für Sonnensystemforschung, Justus-von-Liebig-Weg 3, 37077 Göttingen, Germany

2 School of Mathematical Sciences and Monash Centre for Astrophysics, Monash University, Melbourne, Victoria 3800, Australia 